\title{
On the Picard Group of Enriques Surfaces
}

\author{
Francois R. Cossec
}

Department of Mathematics, University of Michigan, Ann Arbor, MI 48109, USA

\section{Introduction}

An Enriques surface over an algebraically closed field $k$ of characteristic $\neq 2$ is a non-singular projective surface $S$ with $H^{1}\left(S, \mathcal{O}_{S}\right)=H^{2}\left(S, \mathcal{O}_{S}\right)=0$ and $2 K_{S}=0$. The unramified double cover defined by $K_{S}$ is a $K 3$ surface $R$, a non-singular projective surface with $H^{1}\left(R, \mathcal{O}_{R}\right)=0, K_{R}=0$.

Illusie has shown, [I], that the group of divisors modulo numerical equivalence is isomorphic to the Enriques lattice $U \oplus E_{8}(-1)$ where $U$ and $E_{8}(-1)$ denote, respectively, the unique even unimodular lattices of index of inertia $(1,1)$ and $(0,8)$.

The purpose of this note is to use this isomorphism to study the Picard group of $S$. We prove the existence of certain configurations of irreducible curves of arithmetic genus 0 or 1 and deduce from them the existence of certain projective models for $S$ and $R$. For example, we prove the following results:

Theorem 1. Every Enriques surface admits a morphism of degree one onto a surface of degree 10 in $\mathbb{P}^{5}$ with isolated rational double points.

Theorem 2. Every K3 surface which is the étale double cover of an Enriques surface admits a morphism of degree one onto a surface of degree 8 in $\mathbb{P}^{5}$ with isolated rational double points and which is the intersection of three quadric hypersurfaces.

Theorem 3. Every Enriques surface contains three elliptic pencils $\left|2 E_{1}\right|,\left|2 E_{2}\right|$, and $\left|2 E_{3}\right|$ such that $E_{1} E_{2}=E_{2} E_{3}=E_{3} E_{1}=1$.

Theorem 2 is not new, [Co 1, V]. We include it because of the simplicity of the proof given in this paper. Theorem 3 leads to a strong version of the EnriquesArtin theorem, [L].

An Enriques surface is said to be nodal if it contains a nodal curve, i.e. a smooth irreducible rational curve. It is known that the generic Enriques surface is not nodal. This is proved in [L, B-P] via the global Torelli theorem for $K 3$ surfaces. Algebraic properties of the Enriques lattice allow to extend this result to all characteristics and prove the following: 
Theorem 4. The following properties are equivalent:

i) $S$ contains a nodal curve.

ii) $S$ contains an elliptic pencil $|P|$ and a nodal curve $R$ such that $P R=2$.

iii) $S$ contains an elliptic pencil with a reducible fiber.

iv) $S$ admits a morphism of degree two onto a cubic surface in $\mathbb{P}^{3}$.

\section{The Enriques Lattice}

We define the Enriques lattice to be the lattice

$$
E=U \oplus E_{8}(-1),
$$

where $U$ and $E_{8}(-1)$ denote, respectively, the even unimodular lattices of index of inertia $(1,1)$ and $(0,8)$.

This section describes some elementary properties of $E$. We will start by introducing some notations [Ma, Chap. IV].

(1.1) We let $(N, k,(\cdot, \cdot))$ be the triple where

$$
\begin{aligned}
N & =\mathbb{Z}^{11}=\bigoplus_{i=0}^{10} \mathbb{Z} l_{i} \text { for a chosen basis } l_{i}, \\
k & =(-3,1, \ldots, 1) \in N,
\end{aligned}
$$

$(\cdot, \cdot)$ is a bilinear form $N \times N \rightarrow \mathbb{Z}$ given by the formulae

$$
l_{0}^{2}=1, \quad l_{i}^{2}=-1 \quad \text { if } i \geqq 1, \quad l_{i} \cdot l_{j}=0 \text { if } i \neq j .
$$

Then $(N,(\cdot, \cdot))$ is a unimodular lattice of index of inertia $(1,10)$ and $k^{2}=-1$. The orthogonal complement $k^{\perp}$ of $k$ in $N$ is a lattice of index of inertia $(1,9)$. It is even and unimodular hence isomorphic to the Enriques lattice, [S]. We let

$$
\begin{aligned}
R & =\left\{l \in N ; l \cdot k \doteq 0, k^{2}=-2\right\}, \\
I & =\left\{l \in N ; l \cdot k=k^{2}=-1\right\}, \\
P & =\left\{l \in N ; l \cdot k=l^{2}=0\right\} .
\end{aligned}
$$

An element of $R$ is called a root. The map $l \rightarrow k+l$ defines a bijection of $I$ to $P$. Finally, we introduce the following vectors of $k^{\perp}$ :

$$
\begin{aligned}
e_{i} & =-k+l_{i} \text { for } i \geqq 1, \\
d & =10 l_{0}-3\left(l_{1}+\ldots+l_{10}\right), \\
r_{i} & =l_{i}-l_{i+1}=e_{i}-e_{i+1} \text { for } 1 \leqq i \leqq 9, \\
r_{0} & =l_{0}-l_{1}-l_{2}-l_{3}=d-e_{1}-e_{2}-e_{3}, \\
e_{i, j} & =d-e_{i}-e_{j} \text { and } i \neq j \text { and } i, j \geqq 1 .
\end{aligned}
$$

(1.2) The vectors $\left\{r_{i}\right\}$ form a basis of $k^{\perp}$ such that $r_{i}^{2}=-2$ and $r_{i} \cdot r_{j}=0$ or 1 according to whether $r_{i}$ is joined to $r_{j}$ in the diagram

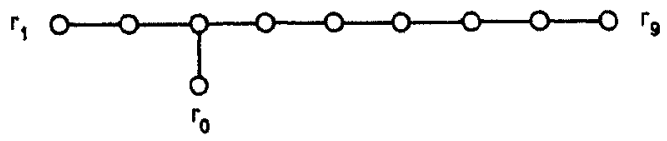


This diagram, usually denoted by $T_{2,3,7}$, defines a lattice $Q_{2,3,7},[\mathrm{Do} 2]$, which is isomorphic to the Enriques lattice. The sublattice of $Q_{2,3,7}$ generated by $\left\{r_{0}, \ldots, r_{8}\right\}$ is isomorphic to $E_{8}(-1)$. Its orthogonal complement is generated by $s_{1}$ and $s_{2}$ where

$$
\begin{aligned}
& s_{1}=3 r_{0}+2 r_{1}+4 r_{2}+6 r_{3}+5 r_{4}+4 r_{5}+3 r_{6}+2 r_{7}+r_{8}, \\
& s_{2}=s_{1}+r_{9}, \\
& s_{1}^{2}=s_{2}^{2}=0, \quad s_{1} \cdot s_{2}=1 .
\end{aligned}
$$

We will identify $k^{\perp}$ and $E$ by means of this orthogonal decomposition of $Q_{2,3,7}$. (1.3) The following properties are immediately checked:

$$
\begin{aligned}
e_{i}^{2} & =0, \quad e_{i} \cdot e_{j}=1 \text { for } i \neq j, \quad i, j \geqq 1, \\
d^{2} & =10, \quad 3 d=e_{1}+\ldots+e_{10}, \quad d \cdot e_{i}=3 \text { for } i \geqq 1, \\
e_{i} \cdot e_{i, j} & =e_{j} \cdot e_{i, j}=2, \quad e_{k} \cdot e_{i, j}=1 \text { for } i, j, k \geqq 1, \quad i \neq j, \quad k \neq i, j .
\end{aligned}
$$

For all $i \geqq 1,\left(d, e_{1}, \ldots, \hat{e}_{i}, \ldots, e_{10}\right)$ is a basis of $E$. For all $i, j, k \geqq 1, i \neq j, k \neq i, j$, $\left(e_{1}, \ldots, \hat{e}_{k}, \ldots, e_{10}, e_{i, j}\right)$ is a basis of $E$.

The dual basis $\left\{b_{j}\right\}$ of $\left\{r_{i}\right\}$ is given by

$$
\begin{aligned}
& b_{0}=d=e_{1}+e_{2}+e_{1,2}, \\
& b_{1}=d-e_{1}=e_{2}+e_{1,2}, \\
& b_{2}=2 d-e_{1}-e_{2}=e_{1}+e_{2}+2 e_{1,2}, \\
& b_{i}=e_{i+1}+\ldots+e_{10} \text { for } i \geqq 3 .
\end{aligned}
$$

(1.4) The linear map $s_{r}: E \rightarrow E$ defined by $s(x)=x+(x r) r$ for each $r \in E$ induces an orthogonal transformation of $O(E)$ which is called the reflection about $r$. The Weyl group of $E$ is the subgroup $W(E)$ of $O(E)$ generated by the reflections about $r_{i}$. The Enriques lattice has the two following properties:

$$
O(E)=W(E) \times\{ \pm 1\},
$$

$R$ is the set of $s$-translates of the $r_{i}$ 's $(s \in W(E))$.

The fundamental chamber $C$ of $E$ is the subset of $L=E \otimes_{\mathbf{z}} \mathbb{R}$ defined by

$$
C=\left\{x \in L ; x \cdot r_{i}>0 \text { for all } i\right\} .
$$

The closure $\bar{C}$ of $C$ in $L$ is the convex polyhedral cone spanned by the vectors $\left\{b_{i}\right\}$. We let

$$
H=\left\{x \in L ; x^{2}>0\right\}
$$

Then $H$ is the disjoint union of two components $H_{1}$ and $H_{2}=-H_{1}$ where $H_{1}$ is the component which contains the fundamental chamber.

The following result can be found in [Bo]. 
Lemma 1.4.1. $\bar{C}$ is a fundamental domain for the action of $W$ on $\bar{H}_{1}$.

As a corollary of this lemma and (1.3), we have

Lemma 1.4.2. Every element $h \in E_{1}$ can be written as a sum $a_{1} f_{2}+\ldots+a_{10} f_{10}$ where the $a_{i}$ 's are positive real numbers and the $f_{i}$ 's satisfy:

$$
f_{i}^{2}=0, \quad f_{1} \cdot f_{3}=f_{2} \cdot f_{3}=2, \quad f_{i} \cdot f_{j}=1 \quad \text { for } \quad\{i, j\} \neq\{1,3\},\{2,3\} \text { and } i \neq j .
$$

A simple computation based on (1.3) and Lemma 1.4.1 leads also to:

Lemma 1.4.3. Any primitive vector of $E$ of length $2 n, 0 \leqq n \leqq 5$, is $O(E)$-equivalent to one of the following vectors:

$$
\begin{aligned}
& n=0: b_{9} ; n=1: b_{8} ; \quad n=2: b_{1}, b_{8}+b_{9}, \\
& n=3: b_{7}, b_{8}+2 b_{9} ; \quad n=4: b_{8}+3 b_{9} ; \quad n=5: b_{0}, b_{7}+b_{9}, b_{8}+4 b_{9} .
\end{aligned}
$$

(1.5) For any $h \in H_{1} \cap E$, we define

$$
\phi(h)=\underset{e \in P}{\operatorname{Min}}|e \cdot h|
$$

Lemma 1.5. $2 \phi(h) \leqq h^{2}$.

Proof. By Lemma 1.4.1, we can assume that $h=n_{0} b_{0}+\ldots+n_{9} b_{9}$ for some positive integers $n_{i}$. Then

$$
\begin{aligned}
h^{2} & =h \cdot\left(n_{0}\left(e_{1}+e_{2}+e_{1,2}\right)+n_{1}\left(e_{1}+e_{1,2}\right)+\ldots+n_{9} e_{10}\right) \\
& \geqq\left(3 n_{0}+2 n_{1}+4 n_{2}+7 n_{3}+6 n_{4}+\ldots+n_{9}\right) \phi(h) .
\end{aligned}
$$

In particular, $h^{2}<2 \phi(h)$ only if $h=b_{9} \notin H_{1}$.

Remark. For every $x \in C \cap E$ with $x^{2} \leqq 10, \phi(x)=1$ or 2 but for $d$ in which case $\phi(d)=3$.

(1.6) A sequence $\left(f_{1}, \ldots, f_{\mathrm{p}}\right)$ of elements $f_{i} \in P$ (respectively $I$ ) is said to be exceptional if $f_{i} \cdot f_{j}=1$ (respectively 0 ) for every $i \neq j$. Note that $p \leqq 10$.

Lemma 1.6.1. $O(E)$ acts transitively on the set of exceptional sequences of vectors of $P$ of length $p \neq 9$. If $p=9$, there are two orbits: the orbits of $\left(e_{1}, \ldots, e_{8}, e_{9}\right)$ and the orbit of $\left(e_{1}, \ldots, e_{8}, e_{1,2}\right)$.

Proof. The map $\left(f_{1}, \ldots, f_{p}\right) \rightarrow\left(-k+f_{1}, \ldots,-k+f_{p}\right)$ defines a 1-1 correspondence between exceptional sequences of vectors of $\boldsymbol{P}$ and exceptional sequences of vectors of $I$, of the same length $p$. Since all primitive vectors of $P$ hence all vectors of $I$, are $O(E)$-equivalent by Lemma 1.4.3, the same proof as in [De, II, Proposition 4] applies to our case.

Lemma 1.6.2. Let $\left(f_{1}, \ldots, f_{10}\right)$ be an exceptional sequence of vectors of $P$ of length 10. Then

i) There exists $d \in E$ such that $3 d=f_{1}+\ldots+f_{10}$.

ii) For any $i, j, i \neq j$, there exists $e_{i, j} \in P$ such that $e_{i} \cdot e_{i, j}=e_{j} \cdot e_{i, j}=2$ and $e_{k} \cdot e_{i, j}=1$ for $k \neq i, j$.

Proof. This follows exactly from (1.3) and Lemmas 1.4.3 and 1.6.1. 


\section{Linear Systems on Enriques Surfaces}

This section describes some basic properties of linear systems on Enriques surfaces. Proofs when omitted can be found in [Co 1].

$S$ will always denote an Enriques surface over an algebraically closed field of characteristic $\neq 2$. If $L$ is an invertible sheaf or a divisor on $S,|L|$ will denote its associated complete linear system.

(2.1) Genus Formula. The arithmetic genus $p_{a}(C)$ of an irreducible curve $C$ on $S$ is given by

$$
p_{a}(C)=1+\frac{C^{2}}{2} \text {. }
$$

In particular, $C^{2} \geqq-2$ and $C$ is a nodal curve, i.e. a smooth irreducible rational curve, if and only if $C^{2}=-2$. An Enriques surface which contains a nodal curve is said to be nodal, unnodal otherwise.

(2.2) Bertini's Theorem [Co 1, Theorem 1.5.1]. Let $L$ be an invertible sheaf on $S$ such that $|L|$ is not empty and without fixed components. Then either:

i) $L^{2}>0$ and there exists an irreducible curve $C$ such that $L \simeq \mathcal{O}_{S}(C)$ and $\operatorname{dim}|L|$ $=p_{a}(C)-1$, or

ii) $L^{2}=0$ and there exist an elliptic pencil $|P|$ and an integer $k$ such that $L \simeq \mathcal{O}_{S}(k P)$ and $\operatorname{dim}|L|=k$.

Remarks. i) Every elliptic pencil $|P|$ on $S$ has exactly two multiple fibres. These are double fibres, $2 E_{1}$ and $2 E_{2}$, such that $K \sim E_{1}-E_{2}$ where " $\sim$ " denotes linear equivalence. Moreover, $E_{1}$ and $E_{2}$ are smooth elliptic curves, rational curves with a node or divisors of type $A_{n}$. We refer to [Mu] for the notion of divisor of canonical type and adopt Rudakov-Safarevic's convention of denoting an indecomposable divisor of canonical type by the corresponding Dynkin diagram, whenever possible.

ii) Conversely, for every indecomposable divisor of canonical type $E$ on $S$, $\operatorname{dim}|E|=1$ or $\operatorname{dim}|2 E|=1$.

Lemma 2.3 [Co 1, Proposition 1.5.2]. Let $L$ be an invertible sheaf on $S$ such that $L^{2}>0$. Then the general member of $|L|$ is irreducible.

Proposition 2.4. Let $D$ be an effective divisor on $S$ such that $D^{2}>0$ and $D C \geqq 0$ for every irreducible curve $C$. Then $|D|$ has no fixed components unless $|D|=|P+R|$ for an elliptic pencil $|P|$ and a nodal curve $R$ such that $P R=2$.

Proof. Let $|D|=|M|+Z$ be the decomposition of $|D|$ into its moving part $|M|$ and its fixed part $Z$. Since $D^{2}>0$, Riemann-Roch implies $|M| \neq \phi$.

Assume $M^{2} \neq 0$. Then (2.2) and Riemann-Roch gives

$$
\frac{M^{2}}{2}=\operatorname{dim}|M|=\operatorname{dim}|D| \geqq \frac{D^{2}}{2} .
$$

Since $D^{2}=D M+D Z=M^{2}+M Z+D Z, M Z \geqq 0, D Z \geqq 0$ it follows that

$$
M Z=D Z=0 \text { hence } Z^{2}=0 .
$$

Hence $Z=0$ by the Hodge index theorem, as wanted. 
Assume $M^{2}=0$. By Lemma 2.3 and Riemann-Roch, $|M|$ is an elliptic pencil $|P|$ and $D^{2}=2$. We can choose a component $R$ of $Z$ such that $P R \geqq 2$. Then

$$
2=D^{2}=D(P+Z)=R P+(Z-R) P+D Z
$$

gives

$$
P(Z-R)=D Z=D R=0, \quad P R=D(P+R)=2 .
$$

By the Hodge index theorem

$$
2\left(2 P R+R^{2}\right)=(P+R)^{2} D^{2} \leqq((P+R) D)^{2}=4
$$

hence

$$
R^{2}=-2, \quad|D|=|P+R| .
$$

The reducibility of $|P+R|$ is proved in the next lemma.

Lemma 2.5. Let $|P|$ be an elliptic pencil and $R$ a nodal curve on $S$ such that $P R=2$. Then

i) $R$ is the fixed part of $|P+R|$.

ii) $\left|P+R+K_{S}\right|$ is irreducible.

Proof. We first prove i). Consider the decomposition $|P+R|=|M|+Z$ of $|P+R|$ into its moving part $|M| \neq \phi$ and its fixed part $Z$.

Assume $M^{2} \neq 0$. Then

$$
M^{2} \leqq M(M+Z)=M(P+R) \leqq(P+R)^{2}=2
$$

shows that $M^{2}=2$ : this is absurd since $\operatorname{dim}|P|=\operatorname{dim}|M|=1$ would imply $|P|=|M|$.

Assume $M^{2}=0$. Then by Lemma 2.3 and Bertini's theorem, $|M|$ is an elliptic pencil $|Q|$ and

$$
2=P(P+R)=P(Q+Z) \geqq P Q
$$

implies $|P|=|Q|, R=Z$ as wanted.

Let us now show that $\left|P+R+K_{S}\right|$ is irreducible. By Proposition 2.4, we can assume, ab absurdo, that there exists elliptic pencil $|Q|$ and a nodal curve $S_{0}$ such that $Q S_{0}=2$ and $\left|P+R+K_{S}\right|=|Q|+S_{0}$. Then

$$
2=P(P+R)=P\left(Q+S_{0}\right) \geqq P Q
$$

implies $|P|=|Q|$ and $R+K_{S} \sim S_{0}$ in contradiction with $\left|R+K_{S}\right|=\phi$ by RiemannRoch.

Remark. An Enriques surface $S$ with an elliptic pencil $|P|$ and a nodal curve $R$ such that $P R=2$ is classically called an Enriques surface of special type. It will be shown in Sect. 4, that this notion coincides with the notion of nodal Enriques surface.

Theorem 2.6. Let $D$ be an effective divisor on $S$ such that $D^{2}>0$ and $D C \geqq 0$ for every irreducible curve $C$. Then

$$
H^{1}\left(S, \mathcal{O}_{S}(-D)\right)=H^{1}\left(S, \mathcal{O}_{S}(D)\right)=0 \text {. }
$$

Proof. If $D$ is an effective divisor such that $D^{2}>0$, Riemann-Roch implies that there exists an effective divisor $\Delta$ such that $\Delta \in\left|D+K_{S}\right|$. Since $K_{S}$ is numerically 
trivial, $D C \geqq 0$ implies $\Delta C \geqq 0$ for every irreducible curve $C$. By Serre duality,

$$
H^{1}\left(S, \mathcal{O}_{S}(-\Delta)\right) \simeq H^{1}\left(S, \mathcal{O}_{\mathrm{S}}(D)\right) \text {. }
$$

Therefore, it is enough to prove the vanishing of $H^{1}\left(S, \mathcal{O}_{S}(-D)\right)$.

If the generic member of $\left|D+K_{S}\right|$ is irreducible, we can conclude by (2.2)i). If the generic member of $\left|D+K_{s}\right|$ is reducible, we can conclude by Proposition 2.4.

Remark. In characteristic 0 , this theorem follows from the vanishing theorem of Zariski-Kawamata.

The following lemma is also an easy consequence of Proposition 2.4 and Lemma 2.5 .

Lemma 2.7. Let $|2 E|$ be an elliptic pencil and $R$ a nodal curve on $S$ such that $E R=n \geqq 2$. Then the generic member of $|E+R|\left(\right.$ or $\left|E+R+K_{S}\right|$ if $\left.n=2\right)$ is irreducible.

(2.8) Pic(S), NS $(S)$, and $\operatorname{Num}(S)$ will denote, respectively, the Picard group, the Neron-Severi group and the group of divisors modulo numerical equivalence. It is well-known that

$$
\begin{aligned}
\mathrm{NS}(S) & \simeq \operatorname{Pic}(S), \\
\operatorname{Num}(S) & \simeq \mathrm{NS}(S) / \text { Torsion } .
\end{aligned}
$$

Moreover, the class of the canonical divisor is the only non-trivial torsion element of $\mathrm{NS}(S)$.

A fundamental result for this paper is the following theorem, [I].

Theorem 2.8. $\operatorname{Num}(S)$ is isomorphic to the Enriques lattice.

(2.9) The elements of Num(S) which are represented by nodal curves are called nodal roots. The set of nodal roots is denoted by $R^{n}$. It lies naturally in $\mathrm{NS}(S)$. We let $W$ be the Weyl group of $\operatorname{Num}(S)$ and $W^{n} C W$ the subgroup corresponding to $R^{n}$. The actions of $W$ and $W^{n}$ on $\operatorname{Num}(S)$ naturally extend to actions on $N S(S)$. We let:

$$
\begin{aligned}
\bar{H}_{+} & =\left\{x \in \mathrm{NS}(S) ; x>0, x^{2} \geqq 0\right\}, \\
\bar{C}^{n} & =\left\{x \in \bar{H}_{+} ; x \cdot r \geqq 0 \text { for every } r \in R^{n}\right\} .
\end{aligned}
$$

Lemma 2.9. For every $e \in \mathrm{NS}(S)$ such that $e>0, e^{2}=0$, there exists a divisor of canonical type $E$ such that $e-E$ is a sum of nodal curve, with non-negative integral coefficients.

Proof. Fix an ample divisor $H$ on $S$. Note that $e H=1$ imply that $e$ is an irreducible curve of arithmetic genus one, hence a divisor of canonical type. Assume now that $e H>1$ and that $e$ is not of canonical type. We can choose a nodal curve $R$ such that $e R<0$ and let $e_{1}=e+(e R) R$. Then $e_{1}^{2}=0$ and $-e_{1}$ is not effective. Therefore, by Riemann-Roch, $e_{1}$ is effective and $e_{1} H<e H$. One concludes by induction on $e H$.

Corollary 2.9. $S$ is an elliptic surface.

We will also need the following result of [Do 2]:

Lemma 2.10. $\bar{C}^{n}$ is a fundamental domain for the action of $W^{n}$ on $\bar{H}_{+}$. 
(2.11) Given an irreducible curve $C$ on $S$ with $C^{2}>0$, we define $\phi(C)$ and $\phi(|C|)$ to be equal to $\phi([C])$ where $[C]$ denotes the class of $C$ in $\operatorname{Num}(S)$ and $\phi$ is the function defined in (1.5). It is clear that

$$
\phi(C)=\underset{E}{\operatorname{Min}}\{E C ;|2 E| \text { is an elliptic pencil }\} .
$$

(2.12) Hyperelliptic Systems. If $C$ is an irreducible curve on $S$ such that $C^{2}>0$ and $\phi(C)=1$, we say that $|C|$ is hyperelliptic. This notion is discussed in [Co 1]. We recall the following results: curve.

i) The generic member of a hyperelliptic system is a smooth hyperelliptic

ii) $|C|$ is hyperelliptic if and only if $|C|$ has base-points.

iii) $|C|$ is hyperelliptic if $C^{2}=2$.

iv) $|C|$ is hyperelliptic if and only if it is of one of the following types:

a) Non-special: $|C|=|p E+F|$ where $p$ is a positive integer and $|2 E||2 F|$ are two elliptic pencils with $E F=1$.

b) Special: $|C|=|(p+1) E+R|$ or $\left|(p+1) E+R+K_{S}\right|$ where $p$ is a positive integer, $|2 E|$ an elliptic pencil and $R$ a nodal curve with $E R=1 .(p \neq 1$ in the first case.)

\section{The Picard Group of $S$}

This section is devoted to some existence theorems of certain linear systems on $S$.

We first note that the following result of Enriques is an immediate consequence of Lemmas 1.4.2 and 2.9.

Theorem 3.1. Pic $(S)$ is generated by the class of nodal curves and irreducible curves of arithmetic genus one.

Theorem 3.2. Let $S$ be an unnodal Enriques surface. Then there exist

i) Ten indecomposable divisors of canonical type $E_{i}$ such that $E_{i} E_{j}=1$ for $i \neq j$.

ii) An irreducible curve $\Delta$ such that $3 \Delta \sim \sum_{i} E_{i}+K_{S}$.

iii) Forty-five divisors of canonical type $E_{i, j}$ for any $\{i, j\}$ with $i \neq j$ such that $E_{i} E_{i, j}=E_{j} E_{i, j}=2, E_{k} E_{i, j}=1$ for $k \neq i, j$ and $\Delta \sim E_{i}+E_{i}+E_{i, j}$.

Proof. This follows directly from Lemmas 2.9, 1.6.2, and Proposition 2.4 applied to any exceptional sequence of isotropic vectors of $\operatorname{Num}(S)$ of length 10 .

Remark. In the notations of Theorem 3.2, $\phi(\Delta)=3$ and $\left\{\Delta, E_{1}, \ldots, E_{10}\right\}$ is a basis of $\operatorname{Pic}(S)$.

Theorem 3.2i) is generalized to the nodal case by the following result:

Theorem 3.3. Let $e_{1}, \ldots, e_{p}$ be some effective divisors on $S$ such that $e_{i}^{2}=0, e_{i} e_{j}=1$ for $i \neq j$. (If $p=1$, we assume that $e_{1}$ defines a primitive vector of $\operatorname{Num}(S)$.) Then there exists a unique set of divisors

$$
E_{1}, \ldots, E_{q}, R_{1,1}, \ldots, R_{1, i(1)}, \ldots, R_{q, 1}, \ldots, R_{q, i(q)},
$$

where $q$ and $i(j)$ are some integers such that $1 \leqq q \leqq p, 0 \leqq i(j)$ for any $1 \leqq j \leqq q$ and $\sum_{j=1}^{q}(1+i(j))=p$, where $\left|2 E_{j}\right|$ are some elliptic pencils and $R_{k, l}$ some nodal curves 
such that

$$
\begin{aligned}
& w\left(e_{1}\right) \approx E_{1}, \\
& w\left(e_{2}\right) \approx E_{1}+R_{1,1} \text { or } E_{2} \text { if } \quad i(1)=0 \\
& \vdots \\
& w\left(e_{p}\right) \approx E_{q}+R_{q, 1}+\ldots+R_{q, i(q)} \text { or } E_{q} \text { if } i(q)=0
\end{aligned}
$$

for some $w \in W^{n}$ (" $\approx$ " denotes numerical equivalence).

The following obvious lemma will be used in the proof of Theorem 3.3:

Lemma 3.3.1. Let $e_{1}, e_{2}, e_{3}$ be three effective divisors on $S$ such that $\left|2 e_{1}\right|$ is an elliptic pencil, $e_{2}^{2}=0, e_{2} e_{3}=1$. Then $e_{1} e_{2}=0$ implies $\left|2 e_{1}\right|=\left|2 e_{2}\right|$.

(3.3.2) Proof of Theorem 3.3. (We will write " $D_{1}=D_{2}$ " instead of " $D_{1} \approx D_{2}$ "): The proof will be by induction on $p$ and based essentially on Lemma 2.10 .

Let us first explain the case $p=1$. By Lemma 2.10, there exists a unique effective divisor $E_{1}$ and $w \in W^{n}$ such that $w\left(e_{1}\right)=E_{1}$. Since $e_{1}^{2}=E_{1}^{2}=0$ and $E_{1} R \geqq 0$ for every nodal curve $R, E_{1}$ is a divisor of canonical type. Since the class of $E_{1}$ in $\operatorname{Num}(S)$ is primitive, $\left|2 E_{1}\right|$ is necessarily an elliptic pencil.

Assume that the theorem holds for $p \geqq 1$ and let $e_{1}, \ldots, e_{p+1}$ be some effective divisors such that $e_{i}^{2}=0, e_{i} e_{j}=1$ for $i \neq j$. By induction, we can assume that $w\left(e_{1}\right), \ldots, w\left(e_{p}\right)$ is as described in Theorem 3.3 for some $w \in W^{n}$. Changing $e_{p+1}$ into $w\left(e_{p+1}\right)$, we can assume $w$ to be the identity.

If $e_{p+1}$ is of canonical type, one simply lets $E_{p+1}=e_{p+1}$.

If $e_{p+1}$ is not of canonical type, we choose a nodal curve $R$ such that $e_{p+1} R<0$ and define

$$
s_{0}\left(e_{p+1}\right)=e_{p+1}+\left(e_{p+1} R\right) R
$$

Then

$$
E_{j} s_{0}\left(e_{p+1}\right)=1+\left(E_{j} R\right)\left(e_{p+1} R\right)=1
$$

unless

$$
E_{j} R=1, \quad e_{p+1}=E_{j}+R \quad \text { (by Lemma 3.3.1) }
$$

in which case $i(j) \neq 0$, since otherwise

$$
0=e_{p+1} R_{j, 1}=1+R_{j, 1} R \neq 0 \text {. }
$$

Therefore, if for some $j, E_{j} s_{0}\left(e_{p+1}\right) \neq 1$, we can assume, after a change of indexation, that $j=q$ and

$$
e_{1}=E_{1}, \quad e_{2}=E_{1}+R_{1,1}, \ldots, e_{p}=E_{q}, \quad e_{p+1}=E_{q}+R .
$$

We now assume that $E_{j} s_{0}\left(e_{p+1}\right)=1$ for all $j=1, \ldots, q$ and we fix an ample divisor $H$.

Assume $R R_{j, k}=0$ for all $j, k$. Then the vectors $e_{1}, \ldots, e_{p}, s_{0}\left(e_{p+1}\right)$ satisfy the same hypothesis as $e_{0}, \ldots, e_{p+1}$ and $H s_{0}\left(e_{p+1}\right)<H e_{p+1}$. By induction on $H e_{p+1}$, we are reduced to the already considered case when $e_{p+1}$ is of canonical type.

Assume $R R_{j, k} \neq 0$ for some $j, k$. We can assume $j=q$. Then we claim that

$$
R R_{q, i(q)}=1 \text { and } R R_{q, j}=0 \text { for } j<i(q) \text {. }
$$


To see this, let $m$ be the integer such that

$$
R R_{q, m} \neq 0 \text { but } R R_{q, n}=0 \text { for } n<m .
$$

Then

$$
R_{q, m} S_{0}\left(e_{p+1}\right)=\left(e_{p+1} R\right)\left(R R_{q, m}\right)<0 .
$$

Define

$$
s_{1}\left(e_{p+1}\right)=s_{0}\left(e_{p+1}\right)+\left(s_{0}\left(e_{p+1}\right) R_{q, m}\right) R_{q, m} .
$$

If

$$
m>1 s_{1}\left(e_{p+1}\right) R_{q, m-1}=s_{0}\left(e_{p+1}\right) R_{q, m}<0
$$

hence we can define $s_{i}\left(e_{p+1}\right)$ for $i=0, \ldots, m$ in such a way that

$$
\begin{gathered}
s_{m-1}\left(e_{p+1}\right) R_{q, 1}=\ldots=s_{0}\left(e_{p+1}\right) R_{q, m}<0, \\
E_{q} S_{m-1}\left(e_{p+1}\right)=\ldots=E_{q} S_{0}\left(e_{p+1}\right)=1
\end{gathered}
$$

and

$$
E_{q} s_{m}\left(e_{p+1}\right)=E_{q} s_{m-1}\left(e_{p+1}\right)+\left(E_{q} R_{q, 1}\right)\left(s_{m-1}\left(e_{p+1}\right) R_{q, 1}\right) \leqq 0 .
$$

Then, by Lemma 3.3.1,

$$
s_{m-1}\left(e_{p+1}\right)=E_{q}+R_{q, 1}, \quad s_{m-1}\left(e_{p+1}\right) R_{q, 1}=-1 .
$$

After $m$ such steps, one finds

$$
e_{p+1}=E_{q}+R_{q, 1}+\ldots+R_{q, m}, \quad R R_{q, m} \neq 0 .
$$

Finally, $m=i(q)$, otherwise

$$
0=e_{p+1} R_{q, m+1}=1+R R_{q, m+1} \neq 0 .
$$

As wanted, we have proved that

$$
e_{p+1}=E_{q}+R_{q, 1}+\ldots+R_{q, i(q)}+R .
$$

The unicity of the $E_{i}$ 's and $R_{j, k}$ 's follows from Lemma 2.10 .

Remarks. i) It follows from Theorem 3.3, that Theorem 3.2i) holds when $S$ is not of special type. However, we recall that the notion of nodal Enriques surface coincide with the notion of Enriques surface of special type.

ii) An immediate corollary of Theorem 3.3 is that an Enriques surface which is not of special type contains two elliptic pencils $|2 E|$ and $|2 F|$ such that $E F=1$. This is well-known, [Co 1, L].

Proposition 3.4. For every elliptic pencil $|2 E|$ on $S$, there exists an elliptic pencil $|2 F|$ such that $E F=1$.

Proof. Since all primitive isotropic vectors of the Enriques lattice are equivalent under its orthogonal group, we can choose an exceptional sequence of isotropic vectors of $N S(S)$ of length 10 , say $\left(e_{1}, \ldots, e_{10}\right)$ such that $E=e_{1}$. By applying Theorem 3.3 to this sequence, we can assume that there exists chain of nodal curve $R_{1}+\ldots+R_{9}$ such that $E R_{1}=0$ and $E R_{i}=0$ for $i>1$. Let $f$ be the fibre of $|2 E|$ which 
contains $R_{2}+\ldots+R_{9}$. Using the fact that rank $(\operatorname{Num}(S))=10$, it is easy to see that $f$ is of type $E_{8}, 2 A_{8}$ or $A_{8}$. In all cases, we denote by $R$ the component of $f$ which is distinct from $R_{2}, \ldots, R_{9}$.

Assume $f$ is of type $E_{8}$ : we obtain the following graph

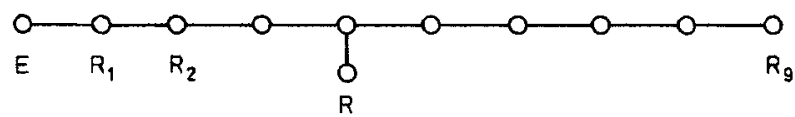

Then $g=2 R+R_{1}+2 R_{2}+3 R_{3}+4 R_{4}+3 R_{5}+2 R_{6}+R_{7}$ is a divisor of type $E_{7}$ such that $E g=1$. This is absurd since $|g|$ is an elliptic pencil hence $g E$ must be even [see remarks following (2.2)].

Assume $f$ is of type $2 A_{8}$ : we obtain the following graph

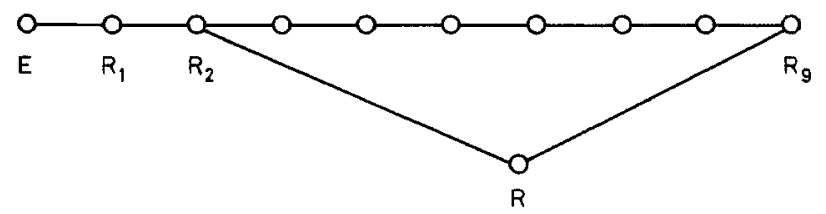

Then $g=2 R_{1}+4 R_{2}+3 R_{3}+2 R_{4}+R_{5}+3 R+2 R_{9}+R_{8}$ is a divisor of type $E_{7}$ such that $g R_{7}=1$, a contradiction.

Finally, if $f$ is of type $A_{8}$, we obtain the following graph

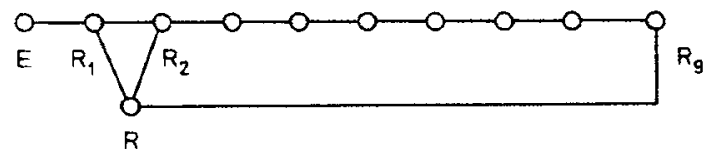

Then $F=R+R_{1}+R_{2}$ defines an elliptic pencil $|2 F|$ such that $E F=1$ as wanted.

Remark. A similar proof would show that given an elliptic pencil $|2 E|$ and a nodal curve $R$ on $S$ such that $E R=1$, there exists an elliptic pencil $|2 F|$ such that $E F=1$ and $F R=0$.

Theorem 3.5. Given an elliptic pencil $\left|2 E_{1}\right|$ on $S$, there exists two elliptic pencils $\left|2 E_{2}\right|$, $\left|2 E_{3}\right|$ such that $E_{1} E_{2}=E_{2} E_{3}=E_{3} E_{1}=1$.

Proof. Given an elliptic pencil $\left|2 E_{1}\right|$ on $S$, Proposition 3.4 implies that we can choose an elliptic pencil $\left|2 E_{2}\right|$ such that $E_{1} E_{2}=1$. By Lemma 1.6.1 there exist an exceptional sequence of isotropic vectors $\left(e_{1}, \ldots, e_{10}\right)$ such that $e_{1}=E_{1}, e_{2}=E_{2}$. Applying Theorem 3.3 to this sequence, we see that we can assume the existence of two chains of nodal curves $R_{1}+\ldots+R_{p}, R_{p+1}+\ldots+R_{8}$ with $0 \leqq p \leqq 8$ and intersection graph

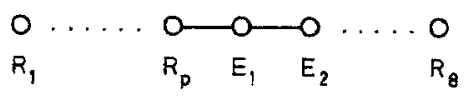

We will prove the theorem when $p=0$. The patient reader could easily supply the details for the other cases.

So let $p=0$. By Lemma 1.6.2, there exists an effective divisor $G$ such that $G^{2}=0$ and defining the following graph:

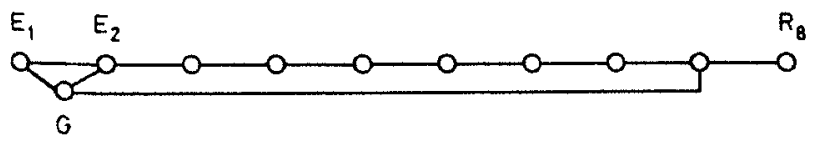


If $G$ is of canonical type there is nothing to prove. So let $R$ be a nodal curve such that $G R<0$.

Assume $R E_{1} \neq 0$. Then $G=R+E_{1}, E_{1} R=1$ (as in the proof of Theorem 3.3) and we have the following graph:

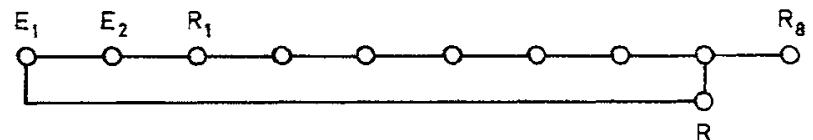

The fibre $g$ of $\left|2 E_{2}\right|$ which contains $R+R_{2}+\ldots+R_{8}$ is necessarily of type $D_{8}$. We let $R_{0}$ be the component of $g$ distinct from $R, R_{2}, \ldots, R_{8}$. We obtain the following diagram

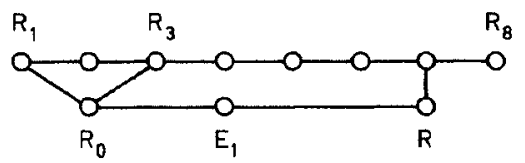

Then $E_{3}=R_{0}+R_{1}+R_{2}+R_{3}$ defines an elliptic pencil $\left|2 E_{3}\right|$ such that $E_{1} E_{2}=E_{2} E_{3}$ $=E_{3} E_{1}=1$ as wanted.

Assume $R E_{1}=0$. As in the proof of Theorem 3.2, one of the following holds

i) $R E_{2}=R R_{1}=\ldots=R R_{5}=R R_{7}=R R_{8}=0, \quad R R_{6}=1$ and $G=E_{2}+R_{1}$ $+\ldots+R_{6}+R$ or

ii) $R R_{1}=\ldots=R R_{6}=R E_{2}=0$.

In case i), $g=3 R+2 R_{8}+4 R_{7}+6 R_{6}+5 R_{5}+4 R_{4}+3 R_{3}+2 R_{2}+R_{1}$ is a divisor of type $E_{8}$ with $g E_{2}=1$, a contradiction.

In case ii) we claim that $R R_{7}=R R_{8}=0$. Otherwise, consider the fibre $g$ of $\left\{2 E_{1} !\right.$ which contains $R+R_{1}+\ldots+R_{8}$. Then $g$ has at least 10 components hence $\operatorname{rank}(\operatorname{Num}(S)) \geqq 11$, a contradiction. Therefore, $R E_{1}=R E_{2}=R R_{i}=0$ for $i \geqq 1$ and changing $G$ into $G+(G R) R$, we can conclude by induction on the degree of $G$ with respect to a fix ample divisor on $S$.

Definition 3.5. A sequence of length $p$ of elliptic pencils $\left(\left|2 E_{1}\right|, \ldots,\left|2 E_{p}\right|\right)$ of $S$ is said to be exceptional if $E_{i} E_{j}=1$ for $i \neq j$.

Remark. Defining $\lambda(S)$ to be the maximum length of exceptional sequence of elliptic pencils on $S, \lambda(S) \leqq 10$ follows trivially from $\operatorname{rank}(\operatorname{Num}(S))=10$ and $\lambda(S) \geqq 3$ follows from Theorem 3.5. Theorem 3.2 implies that $\lambda(S)=10$ if $S$ is unnodal. It is also proved in [Co 2] that $\lambda(S)=10$ for the generic nodal Enriques surface $S$.

Proposition 3.6. $\boldsymbol{S}$ contains a non-hyperelliptic system of arithmetic genus 3 .

Proof. With the notation of (1.3), $b_{1}$ is a vector of length 4 with $\phi\left(b_{1}\right)=2$. Therefore, $S$ contains an effective divisor $D$ with $D^{2}=4$ and $\phi(D)=2$. By Lemma 1.4.1, Proposition 2.4 and Bertini's theorem, the intersection of the $W^{n}$-orbit of $|D|$ with $\bar{C}^{n}$ is an irreducible system with the required properties.

Proposition 3.7. S contains a non-hyperelliptic system $|M|$ of arithmetic genus 6 and $\phi(|M|)=3$.

Proof. Same proof as in Proposition 3.6: one considers $b_{0}$ instead of $b_{1}$. 


\section{Nodal Enriques Surfaces}

This section is devoted to the proof of the following:

Theorem 4.1. The following statements are equivalent:

i) S contains a nodal curve.

ii) $S$ contains an elliptic pencil $|2 E|$ and a nodal curve $R$ with $E R=1$.

iii) S contains two elliptic pencils, $|2 E|$ and $|2 F|$, and a nodal curve $R$ such that $E F$ $=E R=1, F R=0$.

Before giving the proof of Theorem 4.1, let us define $\phi(R)$ for every nodal curve $R$ on $S$ by

$$
\phi(R)=\underset{E}{\operatorname{Min}}\{E R ;|2 E| \text { is an elliptic pencil }\} .
$$

Lemma 4.1.1. $\phi(R)=0$ for every nodal curve $R$ on $S$.

Proof. Let $R$ be a nodal curve on $S$. Assume $\phi(R) \geqq 2$ and let $|2 E|$ be an elliptic pencil with $E R=\phi(R)$. By Lemma 2.7, we can assume $|E+R|$ to be without fixed components. Since $(E+R)^{2}=2(\phi(R)-1)$, Lemma 1.5 and $(2.11)$ show that there exists an elliptical pencil $|2 F|$ with

$$
F(E+R) \leqq \phi(R)-1 \text { hence } F R \leqq \phi(R)-2
$$

contradicting the definition of $\phi(R)$.

In particular, $\phi(R)<2$ so that we can assume $\phi(R)=1$. We conclude by using the Remark following Proposition 3.4.

(4.12) Proof of Theorem 4.1. iii) $\rightarrow$ i) and iii) $\rightarrow$ ii) are obvious. ii) $\rightarrow$ iii) follows from the Remark following Proposition 3.4 hence we only need to prove that i) $\rightarrow$ ii) that is every nodal Enriques surface is of special type. Ab absurdo, we assume $S$ to be nodal but not of special type. We fix a nodal curve $R$ on $S$. By Lemma 4.1.1, we can choose an elliptic pencil $|2 E|$ with $E R=0$. By Proposition 3.4, there exists an elliptic pencil $|2 F|$ such that $E F=1$. Consider the fibre $f$ of $|2 E|$ which contains $R$, since $S$ is not of special type, it is clear that $f$ contains a nodal curve $R^{\prime}$ (not necessarily equal to $R$ ) such that $F R^{\prime}=2$. The classification of hyperelliptic systems [see iii) and iv) of (2.12)] implies that there exist two elliptic pencils $\left|2 E_{1}\right|$ and $\left|2 E_{2}\right|$ with $E_{1} E_{2}=1$ and $\left|F+R^{\prime}\right|=\left|E_{1}+E_{2}\right|$. Then $F E_{1}=F E_{2}=1$ hence, by the Remark following Theorem 3.3, there exists an elliptic pencil $\left|2 E_{3}\right|$ such that $E_{3} F=E_{3} E_{1}=E_{3} E_{2}=1$ hence $E_{3} R^{\prime}=1$ contradicting our assumption.

\section{Projective Models}

This section proves the existence of certain projective models for Enriques surfaces or their associated $K 3$ surfaces using the existence theorems of certain linear systems of Sect. 3.

(5.1) Notations. $C$ will be an irreducible curve on $S$. The map associated to $|C|$ will be denoted by $f_{|C|}$ or simply $f$ if there is no ambiguity:

$$
f: S \rightarrow f(S) \subset \mathbb{P} H^{\circ}\left(S, \mathcal{O}_{S}(C)\right)^{*} \simeq \mathbb{P}^{n}
$$


with $n=p_{a}(C)-1$. We denote by $N_{C}$ the set of nodal curves $R$ on $S$ such that $C R=0$ and let $p: S \rightarrow S_{C}$ be the map contracting the fundamental cycles of $N_{C}$ onto a normal surface $S_{C}$ with isolated rational double points, [A].

\section{(5.2) Genus Two}

(5.2.1) Non-Special Case. $|M|$ will denote a non-special pencil of genus 2 on $S$. There exist two elliptic pencils $\left\{2 E_{1} \mid\right.$ and $\left|2 E_{2}\right|$ such that $E_{1} E_{2}=1$ and $|M|=\left|E_{1}+E_{2}\right|(2.12)$. It is well-known, [Co 1, Do 3], that $|2 M|$ defines a morphism $f$ of degree 2

$$
S \rightarrow Q_{1} \subset \mathbb{P}^{4}
$$

onto a 4-nodal quadric $Q_{1}$ in $\mathbb{P}^{4}$, intersection of the two quadrics of equation

$$
x_{0}^{2}+x_{1}^{2}+x_{2}^{2}=0, \quad x_{0}^{2}+x_{3}^{2}+x_{4}^{2}=0 .
$$

$Q_{1}$ has four singular points of type $A_{1}$. The branch locus of $f$ is composed of the singular locus of $Q_{1}$ and a curve $B \in\left|\mathcal{O}_{Q_{1}}(2)\right|$ which does not intersect the singular locus of $Q_{1}$ and which has admissible singularities, i.e. singularities of type $a_{n}, d_{n}, e_{n}$.

Conversely, the minimal desingularization of $Q_{1}$ branched along the singular locus of $Q_{1}$ and a curve $B$ as described above is an Enriques surface $S$ together with two elliptic pencils $\left|2 E_{1}\right|$ and $\left|2 E_{2}\right|$ such that $E_{1} E_{2}=1$ and such that $\left|2 E_{1}+2 E_{2}\right|$ defines the covering map $S \rightarrow Q_{1}$.

By Corollary 2.9 and Proposition 3.4, this construction yields all Enriques surfaces.

(5.22) Special Case. $|M|$ will now denote a special pencil of genus 2 on $S$. We let $|2 E|$ to be the elliptic pencil and $R$ the nodal curve such that $E R=1$ and $|M|=\left|2 E+R+K_{s}\right|$. Then $|2 M|$ defines a morphism $f$ of degree 2 onto a degenerate 4-nodal quartic $Q_{2}$, [Co 1 , Do 3]:

$$
f: S \rightarrow Q_{2} \subset \mathbb{P}^{4}
$$

which is the intersection of the two quadrics of equation

$$
x_{0}^{2}+x_{1}^{2}+x_{2}^{2}=0, \quad x_{0} x_{4}+x_{3}^{2}=0
$$

$Q_{2}$ has one singular point of type $A_{3}$ and two singular points of type $A_{1}$. The branch locus of $f$ is composed of the singular locus of $Q_{2}$ and a curve $B \in\left|\mathcal{O}_{Q_{2}}(2)\right|$ which does not intersect the singular locus of $Q_{2}$ and has admissible singularities.

Conversely, the minimal desingularization of a double cover of $Q_{2}$ branched along the singular locus of $Q_{2}$ and a curve $B$ as described above is an Enriques surface $S$ together with an elliptic pencil $|2 E|$ and a nodal curve $R$ such that $E R=1$ and such that $|2(2 E+R)|$ defines the covering map $S \rightarrow Q_{2}$.

This construction yields all Enriques surfaces of special type.

\section{(5.3) Genus Three}

Theorem 5.3 [Co 1, V]. Every K3 surface which is the étale double cover of an Enriques surface is birationally equivalent to a complete intersection of three quadric hypersurfaces in $\mathbf{P}^{5}$ which isolated rational double points. 
Proof. Let $p: R \rightarrow S$ be the étale double cover of an Enriques surface $S$. By Proposition 3.6, $S$ contains a non-hyperelliptic system $|M|$ of arithmetic genus 3 . Then $\left|p^{*}(\boldsymbol{M})\right|$ defines the required morphism $R \rightarrow \mathbb{P}^{5}$, see [Co 1, Sect. 8].

\section{(5.3) Genus Four (I)}

(5.3.1) $|C|$ will denote a non-hyperelliptic system of arithmetic genus 4 on $S$. By Lemma 1.4.3, there exist three effective divisors $e_{1}, e_{2}, e_{3}$ such that $e_{i}^{2}=0, e_{i} e_{j}=1$ for $i \neq j$ and $|C|=\left|e_{1}+e_{2}+e_{3}\right|$. Using Lemma 2.10 and Theorem 3.3, $|C|$ is easily seen to be of one of the following types:

1) $\left|E_{1}+E_{2}+E_{3}\right|$ for three elliptic pencils $\left|2 E_{i}\right|$ with $E_{i} E_{j}=1$ for $i \neq j$.

2) $\left|2 E_{1}+E_{2}+R\right|$ for two elliptic pencils $\left|2 E_{1}\right|$ and $\left|2 E_{2}\right|$ and a nodal curve $R$ such that $E_{1} E_{2}=E_{1} R=1, E_{2} R=0$.

3) $\left|3 E_{1}+2 R_{1}+R_{2}\right|$ for an elliptic pencil $\left|2 E_{1}\right|$ and two nodal curves $R_{1}, R_{2}$ such that $E_{1} R_{1}=R_{1} R_{2}=1, E_{1} R_{2}=0$.

Definition 5.3.1. $|C|$ is said to be special if one of the following holds

i) $|C|$ is of type 1) and $E_{1}+E_{2}-E_{3}$ is effective for some elliptic pencils $\left|2 E_{1}\right|$, $\left|2 E_{2}\right|,\left|2 E_{3}\right|$ such that $|C|=\left|E_{1}+E_{2}+E_{3}\right|$.

ii) $|C|$ is of type 2) and $E_{2}-R$ is effective (notation as above).

iii) $|C|$ is of type 3 ) and $E_{1}-R_{2}$ is effective (notation as above).

(5.3.2) We recall the following results, [Co 1, Sect. 7]:

1) $f_{|C|}$ is of degree one if and only if $|C|$ is not special.

2) $|C|$ and $\left|C+K_{S}\right|$ are not simultaneously special.

Proof of 2). The type of $|C|$ is defined by the number of elliptic pencils $|2 E|$ such that $E C=2$. In particular, $|C|$ and $\left|C+K_{S}\right|$ are of the same type. Since type 1) and type 2) are considered in $[\mathrm{Co} 1,(7.8)]$, we will consider only the case when $|C|$ and $\left|C+K_{S}\right|$ are of type 3). Ab absurdo, let us assume that they are special. Let $\left|2 E_{1}\right|, R_{1}$, $R_{2}$ (respectively $\left|2 F_{1}\right|, S_{1}, S_{2}$ ) be an elliptic pencil and some nodal curves such that

$$
\begin{aligned}
|C| & =\left|3 E_{1}+2 R_{1}+R_{2}\right|, \quad E_{1} R_{1}=R_{1} R_{2}=1, \quad E_{1}-R_{2}>0, \\
\left|C+K_{S}\right| & =\left|3 F_{1}+2 S_{1}+S_{2}\right|, \quad F_{1} S_{1}=S_{1} S_{2}=1, \quad F_{1}-S_{2}>0 .
\end{aligned}
$$

Since $\left|2 E_{1}\right|$ (respectively $\left|2 F_{1}\right|$ ) is the only elliptic pencil $|2 E|$ such that $E C=2$ (respectively $E\left(C+K_{S}\right)=2$ ), it follows that $\left|2 E_{1}\right|=\left|2 F_{1}\right|$ hence $E_{1}=F_{1}$ or $E_{1}=F_{1}+K_{s}$.

Assume $E_{1}=F_{1}$. Then $2 R_{1}+R_{2} \sim 2 S_{1}+S_{2}-K_{S}$ hence

$$
R_{1}\left(2 S_{1}+S_{2}+K_{S}\right)=R_{1}\left(2 R_{1}+R_{2}\right)=-3 \text {. }
$$

This is possible only if $R_{1}=S_{1}$ hence $R_{2} \sim S_{2}+K_{S}$ in contradiction with RiemannRoch.

Assume $E_{1}=F_{1}+K_{S}$. Then $2 R_{1}+R_{2} \sim 2 S_{1}+S_{2}$ and as before $R_{1}=S_{1}, R_{2}=S_{2}$ in which case $E_{1}-R_{2}>0$ and $F_{1}-S_{2}=E_{1}+K_{S}-R_{2}>0$ which is absurd since $E_{1}$ and $E_{1}+K_{S}$ do not intersect.

Theorem 5.3.2. Let $S$ be an Enriques surface and $y_{0}, \ldots, y_{3}$ some homogeneous coordinate in $\mathbf{P}^{3}$. Then

i) $S$ is birationally equivalent to a sextic surface in $\mathbb{P}^{3}$ of the form:

$$
y_{0} x_{1} x_{2} x_{3} q(y)+a_{1} y_{0}^{2} x_{1}^{2} x_{2}^{2}+a_{2} x_{1}^{2} x_{2}^{2} x_{3}^{2}+a_{3} x_{2}^{2} x_{3}^{2} y_{0}^{2}+a_{4} x_{3}^{2} y_{0}^{2} x_{1}^{2}=0 \text {. }
$$


ii) $S$ is nodal if and only if $S$ is birationally equivalent to a sextic surface in $\mathbb{P}^{3}$ of the form:

$$
y_{0}^{2} x_{2} x_{3} q(y)+a_{1} y_{0}^{4} x_{2}^{2}+a_{2} y_{0}^{4} x_{3}^{2}+a_{3} y_{0} x_{1} x_{2}^{2} x_{3}^{2}+a_{4} x_{1}^{2} x_{2}^{2} x_{3}^{2}=0
$$

for some linear forms $x_{1}, x_{2}, x_{3}$, some quadratic form $q(y)$ and some $a_{i} \in k$.

Proof. To prove i) (respectively the first part of ii)), one applies Enriques-Artin theorem (see [L, Sect. 3]) to a non-special non-hyperelliptic system of arithmetic genus 4 on $S$ of type 1) (respectively 2)). Such a system exists by Corollary 2.9, Theorem 3.5 (respectively the Remark following Proposition 3.4 and (5.3.2)).

The converse part of ii) follows from [L, Sect. 3].

\section{(5.4) Genus Four (II)}

(5.4.1) We let $\mathscr{C}$ be a projection of $Q=Q_{1}$ or $Q_{2}$ from one of its smooth points. It is a cubic surface in $\mathbb{P}^{3}$ projectively equivalent to one of the following cubics:

$$
\begin{aligned}
& \mathscr{C}_{1}: x_{1} x_{2} x_{3}+x_{2} x_{3} x_{0}+x_{3} x_{0} x_{1}+x_{0} x_{1} x_{2}=0, \\
& \mathscr{C}_{2}: x_{1} x_{3}^{2}+x_{2} x_{3}^{2}+x_{1} x_{2} x_{0}=0, \\
& \mathscr{C}_{3}: x_{3}^{2}+x_{1}^{2} x_{2}+x_{2} x_{3} x_{0}=0 .
\end{aligned}
$$
loci:

The cubic surface $\mathscr{C}_{i}$ are characterized (among cubic surfaces) by their singular

$\mathscr{C}_{1}$ has four singular points of type $A_{1}$.

$\mathscr{C}_{2}$ has one singular point of type $A_{3}$ and two singular points of type $A_{1}$.

$\mathscr{B}_{3}$ has one singular point of type $A_{1}$ and one singular point of type $A_{5}$.

$\mathscr{C}=\mathscr{C}_{1}$ (respectively $\mathscr{C}_{2}, \mathscr{C}_{3}$ ) will be called a non-degenerate (respectively degenerate) 4-nodal cubic.

Lemma 5.4.2. A non-hyperelliptic system $|C|$ of arithmetic genus 4 on $S$ is special if and only if $|C|=|2 M-\Delta|$ for some irreducible pencil $|M|$ of genus 2 and a fundamental cycle $\Delta$ of $N_{M}$, excluding the case where $(|M|, \Delta)$ is of the form $\left(\mid 2 E+R+K_{S}, R\right)$ for an elliptic pencil $|2 E|$ and a nodal curve $R$ such that $E R=1$.

Proof. a) We will first prove that $|2 M-\Delta|$ is a special non-hyperelliptic system of arithmetic genus 4 for every pencil $|M|$ of genus 2 and every fundamental cycle $\Delta$ of $N_{M}$.

Let $|2 M-\Delta|=|N|+Z$ be the decomposition of $|2 M-\Delta|$ into its moving part $|N|$ and its fixed part $Z$. Then

$$
4=\operatorname{dim}|2 M|>\operatorname{dim}|2 M-\Delta| \geqq \frac{(2 M-\Delta)^{2}}{2}=3
$$

implies

$$
3=\operatorname{dim}|2 M-\Delta|=\operatorname{dim}|N|=\frac{N^{2}}{2} .
$$

Then

$$
M(2 M-\Delta)=M(N+Z)=4 \text { hence } M N \leqq 4 \text {. }
$$


By the Hodge index theorem

$$
M^{2} N^{2} \leqq(M N)^{2} \text { hence } M N=4, \quad M Z=0 .
$$

Then

$$
8=N(2 M)=N(N+\Delta+Z) \text { gives } \quad N(\Delta+Z)=2
$$

and

$$
(N+\Delta+Z)^{2}=8 \text { gives }(\Delta+Z)^{2}=-2 .
$$

Since $M(\Delta+Z)=0$ and since $\Delta$ is a fundamental cycle of $N_{M}$, this implies that $Z=0:|2 M-\Delta|$ has no fixed component.

Assume that $|2 M-\Delta|$ is a non-special hyperelliptic system of genus 4 so that there exist two elliptic pencils $|2 E|$ and $|2 F|$ such that

$$
E F=1 \text { and }|2 M-\Delta|=|3 E+F| \text {. }
$$

Then

$$
4=M(2 M)=M(E+F)+2 M E+M \Delta
$$

and the Hodge index theorem implies that $|M|$ is numerically equivalent to $|E+F|$ hence

$$
|M|=|3 E+F-M-\Delta| \approx|2 E+\Delta|
$$

which contradicts the irreducibility of $|M|$ and $\left|M+K_{S}\right|$.

Assume that $|2 M-\Delta|$ is a special hyperelliptic system of genus 4 so that there exist an elliptic pencil $|2 E|$ and a nodal curve $R$ with

$$
E R=1, \quad|2 M-\Delta| \approx|4 E+R| .
$$

As before one proves that $|M|=\left|2 E+R+K_{S}\right|, \Delta=R$.

This last case being excluded, we can now prove that $|C|=|2 M-\Delta|$ is a special non-hyperelliptic system of genus 4 . We will consider only the case when $|M|$ is non-special and leave the special case to the reader. So we let $\left|2 E_{1}\right|$ and $\left|2 E_{2}\right|$ be two elliptic pencils such that

$$
E_{1} E_{2}=1 \text { and }|M|=\left|E_{1}+E_{2}\right| .
$$

Since $|2 M-\Delta|$ is not hyperelliptic

$$
E_{i}(2 M-\Delta)=2-E_{i} \Delta \leqq 2 \text { implies } E_{i}(2 M-\Delta)=2 \text { for } i=1,2 \text {. }
$$

By (5.3.1), $|C|$ is one of the following types:

i) $|C|=\left|E_{1}+E_{2}+E_{3}\right|=\left|M+E_{3}\right|$ where $\left|2 E_{3}\right|$ is an elliptic pencil with $E_{3} M=2$.

ii) $|C|=\left|2 E_{1}+E_{2}+R\right|=\left|M+E_{1}+R\right|$ for a nodal curve $R$ such that $E_{1} R=1$, $E_{2} R=0$.

In case i), $(2 M-\Delta)=\left|M+E_{3}\right|$ hence $E_{1}+E_{2}-E_{3} \sim \Delta>0$. In case ii), $(2 M-\Delta)=\left|M+E_{1}+R\right|$ hence $E_{2}=R+\Delta$. In both cases, $|C|$ is special.

b) Conversely, let $|C|=\left|E_{1}+E_{2}+E_{3}\right|$ be a special non-hyperelliptic system of genus 4 with $|M|=\left|E_{1}+E_{2}\right|=\left|E_{3}+\Delta\right|$ for an effective divisor $\Delta$. We only need to check that $\Delta$ is a fundamental cycle of $N_{M}$. Let $\Delta_{1}$ be the fundamental cycle of $N_{M}$ which contains the support of $\Delta$. Then $\Delta R=-R E_{3} \leqq 0$ for any $R \in N_{M}$ implies that 
$Z=\Delta-A_{1}$ is effective since $\Delta_{1}$ is minimal among all effective cycles $D$ with $D^{2}=-2, D R \leqq 0$ for every $R \in N_{M}$. Now $\left(Z_{1}+\Delta_{1}\right)^{2}=-2$ and $M\left(Z_{1}+\Delta_{1}\right)=0$ implies $Z_{1}=0$ since $\Delta_{1}$ is maximal among all cycles $D$ such that $D^{2}=-2, D M=0$.

Finally, if $|C|=\left|2 E_{1}+E_{2}+R\right|$ (respectively $\left|3 E+2 R_{1}+R_{2}\right|$ ) is a special nonhyperelliptic system of type 2) (respectively 3)) with $E_{2}=R+\Delta$ (respectively $\left.E_{1}=R_{2}+\Delta\right)$ for some effective divisor $\Delta$, one shows as before that $\Delta$ is a fundamental cycle of $N_{M}$ where $|M|=\left|E_{1}+E_{2}\right|$ (respectively $\left|2 E_{1}+R+K_{S}\right|$ ) and $|2 M-\Delta|=|C|$. This concludes the proof.

(5.4.3) We let $(\mid M, \Delta)$ be a pencil $|M|$ of genus 2 together with a fundamental cycle $\Delta$ of $N_{M}$ on $S$.

We assume that $(|M|, \Delta)$ is not of the form $\left(\left|2 E+R+K_{S}\right|, R\right)$ for an elliptic pencil $|2 E|$ and a nodal curve $R$ with $E R=1$. We obtain a commutative diagram

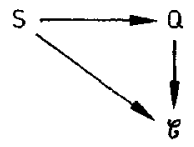

where $S \rightarrow Q$ is the map defined by $|2 M|, S \rightarrow \mathscr{C}$ is the map defined by $|2 M-\Delta|$ and $Q \rightarrow \mathscr{C}$ is the projection from the contracted image of $\Delta$ on $Q$.

The branch locus of $S \rightarrow \mathscr{C}$ is composed of the singular locus of $\mathscr{C}$ and a curve $B \in\left|\mathcal{O}_{\mathscr{E}}(2)\right|$ which does not intersect the singular locus of $\mathscr{C}$ and which has admissible singularities.

Conversely, the minimal desingularization of the double cover of a cubic surface $\mathscr{C}=\mathscr{C}_{i}$ branched along the singular locus of $\mathscr{C}_{i}$ and a curve $B$ as described above is an Enriques surface $S$ together with a special non-hyperelliptic system of type $i$ whose associated morphism is the covering map $S \rightarrow \mathscr{C}$ ([Co 1, Sect. 7]).

We obtain a new characterization of nodal Enriques surfaces.

Theorem 5.4.3. An Enriques surface is a nodal if and only if it admits a morphism of degree two onto a cubic surface in $\mathbb{P}^{3}$.

Proof. Assume that $S$ is an Enriques surface together with a morphism $f: S \rightarrow \mathscr{C}$ of degree two onto a cubic surface. Then $f$ is the map associated to a special nonhyperelliptic system of genus 4, [Co 1], hence $S$ is nodal.

Conversely, assume that $S$ is a nodal surface. In view of the preceding discussion it is enough to show that $S$ contains a pencil $|M|$ of genus 2 and a nodal curve $R_{0} \in N_{M}$ such that $\left(|M|, R_{0}\right)$ is not of the form $\left(\left|2 E+R_{0}+K_{S}\right|, R_{0}\right)$ for an elliptic pencil $|2 E|$ with $E R_{0}=1$.

By Theorem 4.1, we can choose an elliptic pencil $|2 E|$ and a nodal curve $R$ such that $E R=1$. The Remark following Proposition 3.4 allows us to choose another elliptic pencil $|2 F|$ with $E F=1, F R=0$. Using Theorem 3.3, we see that one of the following holds:

i) There exists an elliptic pencil $|2 G|$ such that $G E=G F=1, G R=0$.

ii) There exist two chains of nodal curves $R_{1}+\ldots+R_{p}, R_{p+1}+\ldots+R_{8}$ ( $1 \leqq p \leqq 8)$ with $R_{p}=R$ and intersection graph:

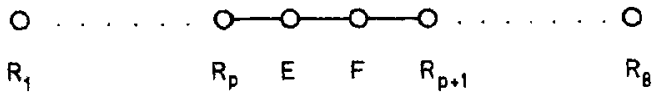


In case i), we take $\left(|M|, R_{0}\right)$ to be equal to $(|F+G|, R)$. In case ii), we take $\left(|M|, R_{0}\right)$ to be equal to $\left(\left|2 E+R_{p}+K_{S}\right|, R_{1}\right)$ if $p \geqq 3$ and $\left(\left|2 E+R_{p}+K_{S}\right|, R_{p+2}\right)$ if $p \leqq 2$.

(5.4.4) Theorem 5.4.3 suggests to introduce a notion of $\mathscr{C}_{i}$-marking similar to the notion of $U$-marking introduced by Dolgachev, [Do 2].

Definition 5.4.4. i) A $\mathscr{C}_{i}$-marked Enriques surface is a pair $(S,|C|)$ of an Enriques surface $S$ and a special non-hyperelliptic system $|C|$ of genus 4 of type i).

ii) An isomorphism between two $\mathscr{C}$-marked Enriques surfaces $(S,|C|)$ and $\left(S^{\prime},\left|C^{\prime}\right|\right)$ is an isomorphism $f: S \rightarrow S^{\prime}$ such that $f^{*} \mathcal{O}_{s^{\prime}}\left(C^{\prime}\right)=\mathcal{O}_{S}(C)$.

We let $U_{i}$ be the open set of $\left|\mathcal{O}_{\mathscr{C}_{i}}(2)\right|$ of curves with admissibles singularities which do not intersect the singular locus of $\mathscr{C}_{i}$. Then $U_{i}$ lies in the set of stable points for the natural action of the automorphism group Aut $\mathscr{C}_{i}$ of $\mathscr{C}_{i}$ on $\left|\mathcal{O}_{\mathscr{V}_{i}}(2)\right|$.

We let $\mathscr{R}_{i}=U_{i} /$ Aut $\mathscr{C}_{i}$. Then $\mathscr{R}_{1}=$ (respectively $\mathscr{R}_{2}, \mathscr{R}_{3}$ ) is an algebraic variety of dimension 9 (respectively 8,7). The generic point of $\mathscr{R}_{i}$ corresponds to a smooth curve of genus 4 together with a non-trivial point of order 2 whose associated symmetric cubic is isomorphic to $\mathscr{C}_{i}$ (see [Ca] for the construction of the symmetric cubic associated to a point of order 2 on a smooth curve of genus 4).

$\mathscr{R}_{i}$ parametrizes the set of isomorphism classes of $\mathscr{C}_{i}$-marked Enriques surfaces.

Assume $\mathscr{R}=\mathbb{C}$ and let $D / \Gamma$ be the period space for Enriques surfaces constructed by Horikawa [Ho]. There is a forgetful map which to a $\mathscr{C}$-marked Enriques surface $(S,|C|)$ associates the period of $S$.

Theorem 5.4.5. The forgetful map $\mathscr{R}=\mathscr{R}_{1} \amalg \mathscr{R}_{2} \amalg \mathscr{R}_{3} \rightarrow D / \Gamma$ is a quasi-finite morphism of algebraic varieties whose image is the irreducible variety of dimension 9 which parametrizes the periods of nodal Enriques surfaces.

Proof. The map $P: \mathscr{R} \rightarrow D / \Gamma$ is shown to be quasi-finite as in [Do 2, Theorem 2.3]. Since an Enriques surface admits a $\mathscr{C}$-marking if and only if it is of special type, the image of $P$ is the irreducible variety of dimension 9 parametrizing the periods of Enriques surfaces of special type (or equivalently the periods of nodal Enriques surfaces).

Remark. The structure of the map $\mathscr{R} \rightarrow D / \Gamma$ is related to the structure of the automorphism groups of nodal Enriques surfaces. We hope to come back to this question in a paper with I. Dolgachev.

\section{(5.5) Genus Six}

The main result of this section is the following:

Theorem 5.5. Every Enriques surface $S$ admits a morphism of degree one onto a surface of 10 in $\mathbb{P}^{5}$ with isolated rational double points.

This will follow from Proposition 3.7 and

Theorem 5.5 bis. Let $C$ be an irreducible curve on $S$ with $C^{2}=10$ and $\phi(C)=3$. Then its associated map $f=f_{|C|}$

$$
f: S \rightarrow f(S) \subset \mathbb{P H}^{0}\left(S, \mathcal{O}_{S}(C)\right)^{\mathfrak{v}} \simeq \mathbb{P}^{5}
$$

is a morphism of degree one onto a surface with isolated rational double points. 
It was proved in [Co 1, Sect. 6] that $f$ is a morphism of degree one onto a surface of degree 10 in $\mathbb{P}^{5}$. So we only need to check that $f(S)$ is normal. The normality of $f(S)$ implies that $f$ factors through $S_{C}$ and Zariski's main theorem implies that $S_{C}$ is isomorphic to $f(S)$.

The idea of the proof will be to combine the following two lemmas:

Lemma 5.5.1 [Co 1, Sect. 8]. Let $|M|$ be a non-hyperelliptic system of genus 3 on S. Then $f_{|M|} \times f_{|M+K|}$ is a morphism of degree one onto a surface with isolated rational double points.

Lemma 5.5.2. Let $C$ be an irreducible curve on $S$ with $C^{2}=10$ and $\phi(C)=3$. Let $|2 E|$ be an elliptic pencil with $E C=3$. Let $|M|$ be the moving part of $|C-E|$. Then

i) $|M|$ is a non-hyperelliptic system of genus 3.

ii) $|C|=|M+E|$ or $|C|=\left|M+E+R_{1}+\ldots+R_{p}\right|$ where $R_{1}+\ldots+R_{p}$ is a chain of nodal curves with intersection graph

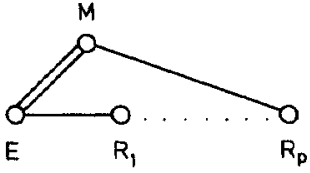

Proof. Let $|C-E|=|M|+F$ be the decomposition of $|C-E|$ into its moving part $|M|$ and its fixed part $F$. By Riemann-Roch,

$$
\frac{M^{2}}{2}=\operatorname{dim}|M|=\operatorname{dim}|C-E| \geqq \frac{(C-E)^{2}}{2}=2 .
$$

By the Hodge index theorem

$$
40 \leqq M^{2} C^{2} \leqq(M C)^{2} \text {. hence } M C \geqq 7 .
$$

Since $E C=3$, we get $M C=7, F C=0, M^{2}=4$. Assume that $|M|$ is hyperelliptic and let $e_{1}, e_{2}$ be two effective divisors such that $e_{1} e_{2}=1, e_{1}^{2}=0, e_{2}^{2}=0,|M|=\left|2 e_{1}+e_{2}\right|$. Then

$$
10=C^{2} \geqq C\left(2 e_{1}+e_{2}+E\right) \geqq 4 \phi(C)=12
$$

is absurd. Therefore, $|M|$ is not hyperelliptic and $E C=3$ implies that $E M=2$ or 3 .

Assume $E M=3$. Then $|C|=|E+M|$ by Riemann-Roch. This is case i).

Assume $E M=2$. Then $E F=1, M F=1, F^{2}=-2$, and $F C=0$. Choose some nodal curves $R_{1}$ and $R_{p}$ of $F$ such that $E R_{1}=M R_{p}=1$. There is a chain of nodal curves of $F$, say $R_{1}+\ldots+R_{p}$, connecting $R_{1}$ to $R_{p}$. Riemann-Roch theorem implies that

$\operatorname{dim}\left|E+R_{1}+\ldots+R_{p}+M\right|=5$ hence $F=R_{1}+\ldots+R_{p} \quad$ and we are in case ii).

\section{(5.5.3) Proof of Theorem 5.5}

Step 1 (keeping the notations of 5.5.2). i) Let $D$ be an effective divisor with $D^{2}=-2$, $D M=0$. Then $D C \leqq 2$.

ii) Let $R$ be a nodal curve such that $R C=2$. Then $f$ restricted to $R$ is of degree one onto a conic. 
Proof. i) By the Hodge index theorem

$$
20=(M-D)^{2} C^{2} \leqq(M-D) C^{2}
$$

hence $(M-D) C \geqq 5$. Since $C \sim E+Z+D+(M-D), C^{2}=10$ and $E C=3$, it follows that $D C \leqq 2$.

ii) Assume that $f(R)$ is not a conic. Then $f(R)$ is a line and

$$
\operatorname{dim}|C-R|=\operatorname{dim}|C|-2=3 \text {. }
$$

Let $|C-R|=|N|+F$ be the decomposition of $|C-R|$ into its moving part $|N|$ and its fixed part $F$. Then $N^{2}=6$ and $C N \leqq 8$. By the Hodge index theorem

$$
60=N^{2} C^{2} \leqq(N C)^{2} \leqq 64
$$

hence $N C=8, N(R+F)=2,(R+F)^{2}=0, C(R+F)=2$ contradicting $\phi(C)=3$.

We let $f=\phi \circ \theta$ be the factorization of $f$ through $S_{C}$

$$
f: S \stackrel{\theta}{\longrightarrow} S_{C} \stackrel{\phi}{\longrightarrow} f(S) \subset \mathbb{P}^{5} \text {. }
$$

Step 2. $\phi$ is injective.

Proof. It is immediate to check that $H^{1}\left(S, \mathcal{O}_{S}(C-E)\right)=0$. This gives a short exact sequence

$$
H^{0}\left(S, \mathcal{O}_{S}(C)\right) \rightarrow H^{0}\left(E, \mathcal{O}_{E}(C)\right) \rightarrow 0 .
$$

Since $E C=3$, it follows that $\phi$ restricted to $\theta(E)$ is an isomorphism onto a plane cubic.

Assume that $x$ and $y$ are too distinct closed points of $S$ not on a same fundamental cycle of $N_{C}$ and such that $f(x)=f(y)$. By an argument similar to the one given in Lemma 5.2.3 of [Co 1], we can assume that $x, y$ do not lie on any nodal curve of $N_{C}$. Since $|M|$ has no base-points and $|C|=|E+F+M|, x \in E$ if and only if $y \in E$. Since we have just proved that $\phi$ restricted to to $\theta(E)$ is injective, it is clear that we can also assume that $x$ and $y$ do not belong to $E+E^{\prime}$.

Since the curves of $|M|$ and $\left|M+K_{S}\right|$ separate the points of $S_{M}$, by Lemma 5.5.1, there exists a chain of nodal curves $D=R_{1}+\ldots+R_{n}$ such that $x \in R_{1}, y \in R_{n}$, and $M D=0$. By Step $1, D C=0,1$ or 2 . Since $x, y$ do not belong to any nodal curve of $N_{c}, D C=1$ or 2 .

Assume that $D C=2$ and $n>2$. Then $C R_{1}=C R_{n}=1$ and $f$ restricted to $R_{1}$ (respectively $R_{n}$ ) is a morphism of degree one onto a line $l_{1}$ (respectively $l_{n}$ ) and $l_{1} \cap l_{n}=f\left(R_{2}+\ldots+R_{n-1}\right)$. Clearly $\phi$ restricted to $\theta(D)$ is injective, contradicting our assumption.

The other cases $D C=1$ and $D C=2, n \leqq 2$ is handled similarly.

Step 3. Let $p$ be a closed point of $S$ such that there exists a curve $R \in N_{M}$ such that $p \in R$ but there exists no curve $R^{\prime} \in N_{c}$ with $p \in R^{\prime}$. Then $f(p)$ is a smooth point of $f(S)$.

Proof. By Step 1, there is a nodal curve $R$ with $p \in R, R M=0$, and $C R=1$ or 2 .

Assume that $C R=1$. Let $|C-R|=|N|+F$ be the decomposition of $|C-R|$ into its moving part $|N|$ and its fixed part $F$. By Step $2, N^{2}=6$ and by the Hodge index theorem

$$
60=N^{2} C^{2} \leqq(N C)^{2} .
$$


Since $N C \leqq 9$, this implies $N C=8$ or 9. Assume $N C=8$, then $N(R+F)=2$, $(R+F)^{2}=0$, and $C(R+F)=2$ contradicting $\phi(C)=3$. Therefore, $N C=9$, $N(R+F)=3,(R+F)^{2}=-2, C(R+F)=-1$. Since $C F=0$ and $p$ does not belong to a nodal curve of $N_{C}, p \notin F$ and $p$ is a smooth point of $R+F$. One easily checks that $|N|$ is not hyperelliptic. Since $f$ restricted to $R$ is an isomorphism onto a line and since $\mathrm{p}$ is not a base-point of $|N+F|,|C|=|R+(N+F)|$ clearly implies that $f(p)$ is a smooth point of $f(S)$.

Assume $C R=2$. Let $|C-R|=|N|+F$ as before. Then $N^{2}=4$ by Step 1 and $N C=7$ or 8 by the Hodge index theorem. Assume $N C=8$, then $N(R+F)=4$, $(R+F)^{2}=-2, C(R+F)=2$. Since $C F=0, p \notin F$. One checks that $|N|$ is not hyperelliptic and concludes as before. Assume $N C=7$, then $N(R+F)=3$, $(R+F)^{2}=0, C(R+F)=3$, and $|N|$ is not hyperelliptic. Let $|2 E|$ be an elliptic pencil and $Z$ an effective divisor such that $R+F=E+Z$. Since $|N|$ is not hyperelliptic, $N E \geqq 2$ and $|C|=|E+N|, Z=0$ by Riemann-Roch. Therefore, $2=C R=R N$ implies that $|R+N|$ is a non-hyperelliptic system. If $p \notin F, p$ is a smooth point of $R$ and not a base-point of $|F+N|$. If $p \in F, p$ is a smooth point of $F$ and not a base-point of $F$ and not a base-point of $|R+N|$. Then $|C|=|F+R+N|$ clearly implies that $f(p)$ is a smooth point of $f(S)$.

Step 4. Let $p$ be a closed point of $S$ which does not belong to any curve of $N_{c}$. Assume that $f(p)$ is a singular point of $f(S)$. Then the generic member of $|C-p|$ is smooth.

Proof. By Lemma 5.5.1 and Step 3, we can assume that $p \in E+E^{\prime}$, say $p \in E$. Since $|M|$ has no base-points, the multiplicity of $f(S)$ at $f(p)$ is the multiplicity of $f(E)$ at $f(p)$. Since $f(E)$ is a plane cubic image of a divisor of type $A_{n}$, this multiplicity is equal to 2 . Clearly $f(S)$ will be smooth in codimension one. It follows that the generic member of $|C-p|$ is smooth outside $p$. Otherwise, a generic 2-dimensional linear system of hyperplane through $f(p)$ would define an inseparable cover of $\mathbb{P}^{2}$ of degree $\operatorname{deg} f(X)-2=8$, contradicting $\operatorname{char}(k) \neq 2$.

Let us check that the generic member of $|C-p|$ is smooth at $p$. Since $p \notin E^{\prime}+F$, it is enough to check that the generic member of $\left|M+K_{S}-p\right|$ is smooth at $p$. If the generic member of $\left|M+K_{S}-p\right|$ is irreducible this is clear since $E M=2$ or 3 and $E$ is singular at $p$. So we can assume that the generic member of $\left|M+K_{S}-p\right|$ is reducible. Since $\operatorname{dim}\left|M+K_{S}\right|=2$ the moving part of $\left|M+K_{S}-p\right|$ is a complete system of dimension 1 . So let

$$
\left|M+K_{s}-p\right|=Z+|N|
$$

for an effective divisor $Z>0$ and a pencil $|N|$ of genus 1 or 2 .

Assume first that $|N|$ is an elliptic pencil. Then $C M=7$ and $C N \geqq 6$ implies $C N=6$ and $C Z=1$. Since $|N|$ has no base-point, $p \in Z$. Since $p$ does not belong to any curve of $N_{c}, p$ is a smooth point of $Z$ hence a smooth point of the generic member of $Z+|N|$.

Assume that $|N|$ is a pencil of genus 2 . As before, we see that $p$ is a smooth point of the generic member of $Z+|N|$ unless $p \in Z$ and $p$ is a base-point of $|N|$. If $\left|N+E^{\prime}\right|$ is not hyperelliptic, we are done since $F+Z+\left|N+E^{\prime}\right|$ is a subsystem of $|C-p|$ where $p$ is a smooth point of $F+Z$ and $\left|N+E^{\prime}\right|$ has no base-point. Therefore, we 
can assume that $\left|N+E^{\prime}\right|$ is hyperelliptic or equivalently $E N=1$. If $F \neq 0, E R_{1}=1$, and $\left(F-R_{1}\right)+Z+\left|N+E^{\prime}+R_{1}\right|$ is a subsystem of $|C-p|$ which is smooth at $p$ because $p$ is a smooth point of $\left(F-R_{1}\right)+Z$ and $\left|N+E^{\prime}+R_{1}\right|$ has no base-point. Therefore, we can assume that $F=0$ hence $E M=3$. It is easy to check that $|M|=|N+Z|$ is of one of the following types:

a) $|M|=\left|E+G+S_{1}+\ldots+S_{q}\right|$ where $|2 G|$ is an elliptic pencil and $S_{1}, \ldots, S_{q}$ some nodal curves with intersection graph

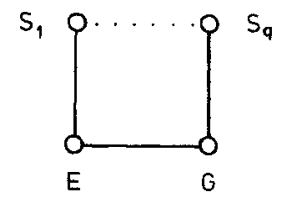

b) $|M|=\left|2 E+R_{1}+R_{2}+K_{S}\right|$ where $R_{1}$ and $R_{2}$ are nodal curves with intersection graph

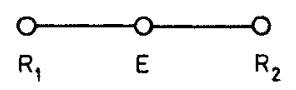

In both cases $E M \neq 3$, contradiction.

Step 5. $\phi$ is an isomorphism.

Proof. One proves as in Lemma 5.3.5 of [Co 1] that $f(Z)$ is a rational double point of $f(S)$ for every fundamental cycle $Z$ of $N_{c}$. So it will be sufficient to check that $f(p)$ does not belong to the singular locus of $f(S)$ for every closed point $p$ which does not belong to any curve of $N_{C}$. Ab absurdo, let $p$ be a point which does not belong to any curve of $N_{C}$ and such that $f(p)$ is a singular point of $f(S)$. By Step 4, the generic member of $|C-p|$ is smooth. We can assume that the restriction $g$ of $f$ to $\gamma$ is injective and that $g(\gamma)$ is smooth outside $f(p)$. By assumption, the tangent map to $g$ is not injective at $p$ hence

$$
H^{1}\left(\gamma, \mathcal{O}_{\gamma}(C-2 p)\right) \simeq H^{1}\left(\gamma, \mathcal{O}_{\gamma}\left(2 p+K_{s}\right)\right) \neq 0 .
$$

Let $q_{1}, q_{2} \in \gamma$ be such that $q_{1}+q_{2} \in\left|\mathcal{O}_{\gamma}\left(2 p+K_{S}\right)\right|$. The fact that $\left|\mathcal{O}_{\gamma}(C)\right|$ has no basepoints implies that $q_{1} \neq p, q_{2} \neq p$. The injectivity of $g$ implies $q_{1}=q_{2}$. Then

$$
H^{1}\left(\gamma, \mathcal{O}_{\gamma}\left(C-2 q_{1}\right)\right) \simeq H^{0}\left(\gamma, \mathcal{O}_{\gamma}\left(2 p+K_{S}\right)\right) \neq 0
$$

implies that $g(\gamma)$ is singular at $f\left(q_{1}\right) \neq f(p)$ a contradiction. This concludes the proof of Theorem 5.5 bis.

Acknowledgements. I would like to thank I. Dolgachev, W. Lang, and S. Mukaï for helpful discussions and the Max-Planck-Institut and the Tata Institute for providing stimulating atmospheres for writing this paper.

\section{References}

[A] Artin, M.: Some numerical criteria for contractability of curves on algebraic surfaces. Am. J. Math. 84, 485-496 (1982)

[Bo] Artin, M.: On isolated singularities of surfaces. Am. J. Math. 88, 129-136 (1966)

[Bo] Bourbaki, N.: Groupes et algèbres de Lie. Paris: Hermann 1968

[B-P] Barth, W., Peters, C.: Automorphisms of Enriques surfaces. Invent. Math. 73, 383-411 (1983) 
[Ca] Catanese, F.: On the rationality of certain moduli spaces related to curves of genus 4, Algebraic Geometry, Proceedings Ann Arbor (1981). Lect. Notes Math., Vol. 1008. Berlin, Heidelberg, New York, Tokyo: Springer 1983

[Co 1] Cossec, F.: Projective models of Enriques surfaces. Math. Ann. 265, 283-334 (1983)

[Co 2] Cossec, F.: Reye congruences. T.A.M.S. 280, 737-751 (1983)

[De] Demazure, M., Pinkham, M., Teissier, B.: Séminaire sur les singularités des surfaces. Notes Math., Vol. 777. Berlin, Heidelberg, New York: Springer 1980

[Do 1] Dolgachev, I.: On automorphisms of Enriques surfaces. Invent. Math. 76, 163-177 (1984)

[Do 2] Dolgachev, I.: Weyl groups and Cremona transformations. Proc. Symp. Pure Math. 40, Part I (1983)

[Ho] Horikawa, E.: On the periods of Enriques surfaces. I. Math. Ann. 234, 73-108 (1978)

[I] Illusie, L.: Complexe de de Rham-Witt et cohomologie cristalline. Ann. Sci. Ec. Norm. Super. 12, 501-661 (1979)

[L] Lang, W.E.: On Enriques surfaces in characteristic p. I. Math. Ann. 265, 45-65 (1983)

[Ma] Manin, Y.: Cubic Forms: algebra, geometry, arithmetic. Amsterdam: North-Holland 1974

[Mu] Mumford, D.: Enriques classification of surfaces in char. p. Part I. Global Analysis. Princeton: University Press 1969

[S] Serre, J.P.: Cours d'arithmetique. Paris: Presses Universitaires de France

[V] Verra, A.: On Enriques surfaces as a four-fold cover of $\mathbb{P}^{2}$. Math. Ann. 266, 241-250 (1983)

Received April 16, 1984; in revised form October 10, 1984 